\title{
On the Decreasing Significance of Large Standard Cells in Technology Mapping
}

\author{
Jae-sun Seo, Igor Markov, Dennis Sylvester, and David Blaauw \\ Department of EECS, University of Michigan, Ann Arbor, MI 48109 \\ \{jseo,imarkov,dmcs,blaauw\}@umich.edu
}

\begin{abstract}
Technology scaling reduces gate delays while wire delays may increase. Our work studies the interaction of this phenomenon with technology mapping and its impact on modern EDA flows. In particular, we demonstrate that the use of larger standard cells increases the number of long wires and may undermine circuit delay optimization at $65 \mathrm{~nm}$ and below. Experiments with $130 \mathrm{~nm}, 90 \mathrm{~nm}, 65 \mathrm{~nm}$, and $45 \mathrm{~nm}$ industrial CMOS technology suggest that limiting the use of larger standard cells in technology mapping becomes more effective at $65 \mathrm{~nm}$ and $45 \mathrm{~nm}$ node, resulting in up to $12 \%$ improvement in critical path delay on large benchmark circuits.
\end{abstract}

\section{INTRODUCTION}

Over several decades, technology mapping has been extremely useful for reducing the device area of complex logic. Furthermore, recent research in Boolean matching [12] accomplished dramatic efficiency improvements for function matching, facilitating new technology mapping algorithms that can deal with 10-input gates. However, extending these algorithms with proper models of circuit delay and validating them with respect to recent technology nodes remains a major research challenge.

While technology mapping seeks to minimize device count, the bulk of critical path delay has shifted from gates to wires in the last 5 years. In particular, the number of repeaters required is exponentially increasing with each technology step [3-4], and $10 \sim 15 \%$ of gates in large microprocessor chips are buffers that break down long interconnects. Extensive literature exists on optimal buffering [5-7] that employs fairly accurate delay modeling, but does not attempt logic restructuring.

Our work is motivated by the apparent dichotomy between (1) the literature on buffer insertion that improves circuit performance by adding a large number of one-input oneoutput gates (buffers and inverters) that do not perform any logic operation, and (2) the literature on functional technology mapping, which clusters logic into 5-15 input gates, improving area, but does not evaluate overall circuit performance with respect to current technology nodes.

Previous literature [8-11] suggests that technology mapping must interact with placement of the standard cells and use accurate interconnect models for performance optimization. These works improved the critical delay through either integration of layout information in early logic synthesis stage [8-9] or iterative re-synthesis with placement information [10-11], but they have not considered the impact of technology mapping on global buffer counts and the overall circuit performance after place-and-route optimizations.

This paper proposes to, ironically, undo technology mapping for high-speed designs through reducing the wire delay components in the critical path of large circuits. Foregoing aggressive technology mapping and using a large number of standard cells (but of smaller size) will eliminate the need for excessive buffers during post-placement timing optimization. The discussions and experiments in this work also consider coupling capacitance between adjacent wires which dominates the wire capacitance in most advanced technologies, and we attempt to reduce the parallel run length of neighboring wires.

Recent work [15] points out that conglomerating small cells into a large cell may produce non-monotonic interconnects which adversely affect delay and routability as illustrated in Figure 1. By limiting the use of large standard cells, our approach inherently blocks the occurrence of this disadvantageous technology mapping, and results in a number of shorter monotonic wires.

Our considerations and conclusions are intended for ASIC/SoC designs rather than FPGA designs. Since programmable interconnect is uniformly buffered and has linear delay in FPGA designs, wire delays do not significantly depend on whether long nets are broken into shorter segments. On the other hand, technology mapping

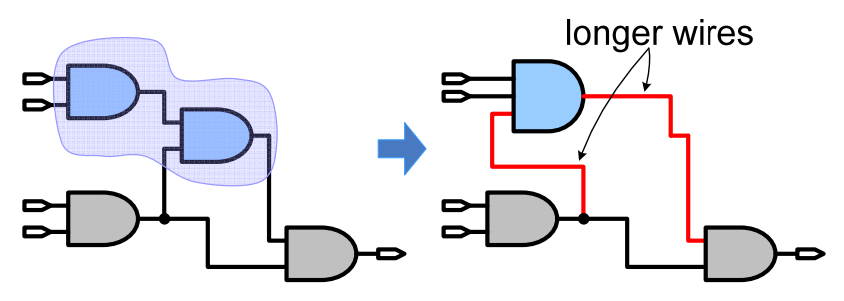

Figure 1: Indiscriminate technology mapping may produce longer wires, adversely affecting delay and routing congestion. 
into LUTs is an important and difficult task. Therefore, in FPGAs, technology mapping still remains useful.

The remainder of this paper is organized as follows. In section 2, a "clean" experiment is analyzed with a simple combinational logic block and a long wire. Section 3 evaluates the impact of standard cell libraries on circuit delay, shows the experimental results, and explains the observations. Section 4 summarizes this paper.

\section{ANALYSIS OF SINGLE PATHS}

As a proof of concept, we conducted a simple experiment as depicted in Figure 2. As a baseline for comparison, 16 3input NAND gates drive a M5 minimum-pitch $5 \mathrm{~mm}$ wire, which is optimally repeated in $65 \mathrm{~nm}$ technology. Traditionally, we would have the combinational logic placed in a denser cluster for minimum area as shown in Figure 2(a). Instead, however, we spread out the 16 NAND gates regularly along the interconnect to implement the logic and also serve as repeaters in Figure 2(b). These distributed NAND gates eliminate long wires and the need for repeaters, resulting in actually better performance. This effect could be exploited by decomposing the logic into more gates, i.e., undoing technology mapping. In Figure 2(c), the long wire is divided more finely with more logic gates (24 2-input NANDs) for the same functionality. Note that, in Figure 2(a)-(c), the inputs of the NAND gates which are not in the critical path are tied to Vdd for worst-case rising delays.

HSPICE simulation is done with industrial $65 \mathrm{~nm}$ CMOS technology, where all three schemes are swept with sizing, and the optimal energy versus delay results are shown in Figure 3. Comparing to scheme (a) at iso-energy of $1.1 \mathrm{pJ}$, scheme (b) achieves 13\% delay reduction and scheme (c) achieves $18 \%$ delay reduction. Overall, (c) improves the energy-delay curve of (a) by a significant amount. At these delay points, Figure 4 shows a delay breakdown of the three

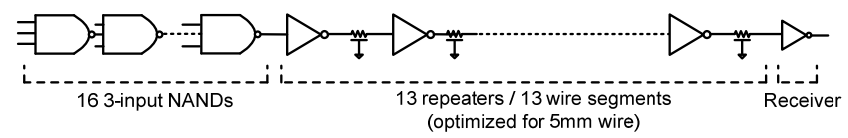

(a)

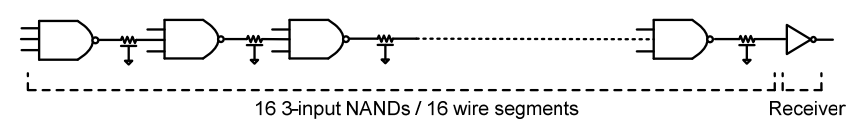

(b)

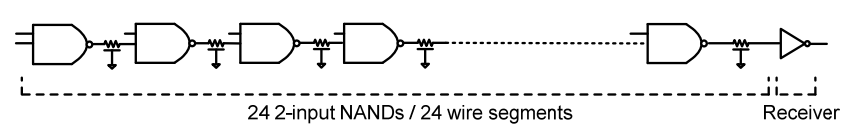

(c)

Figure 2: Three schemes for comparison of single paths (a) Logic block (16 3-input NANDs) driving an optimally repeated $5 \mathrm{~mm}$ wire (b) 163 -input NANDs are placed along the wire (c) 16 3-input NANDs are decomposed into 24 2input NANDs and placed along the wire.

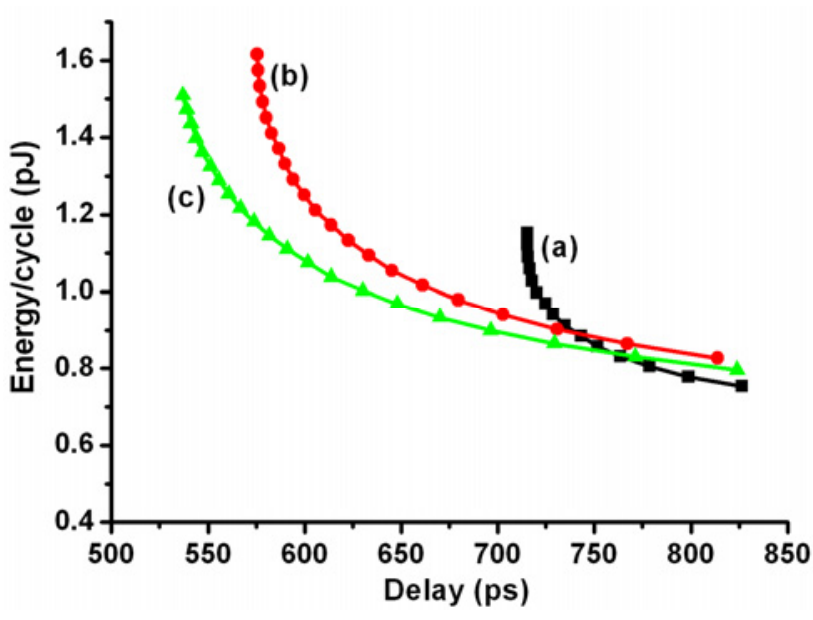

Figure 3: Energy versus delay comparison for the three different schemes in Figure 2.

schemes. By spreading out the NAND gates in (b), logic gate delay is increased since the load capacitance of the NAND gates is increased due to wires, but the repeater delay portion is eliminated and the overall delay is reduced by $13 \%$. Through undoing technology mapping in (c), wire delay is further reduced due to fine chopping of wires and gate delay also slightly decreased due to reduced load capacitance. Interestingly, if long wires are present in the circuit delay, spreading out the gates and using more gates to implement a given logic could actually improve delay since they convert wire delay back to logic delay.

One possible drawback of this approach is that the inputs to the NAND gates in the middle of the wire have to be routed to the intermediate placement locations, but the surrounding logic gates could be restructured and placed nearby the middle of the wire. We scrutinized this in the following section by performing synthesis, placement and routing on large benchmarks.

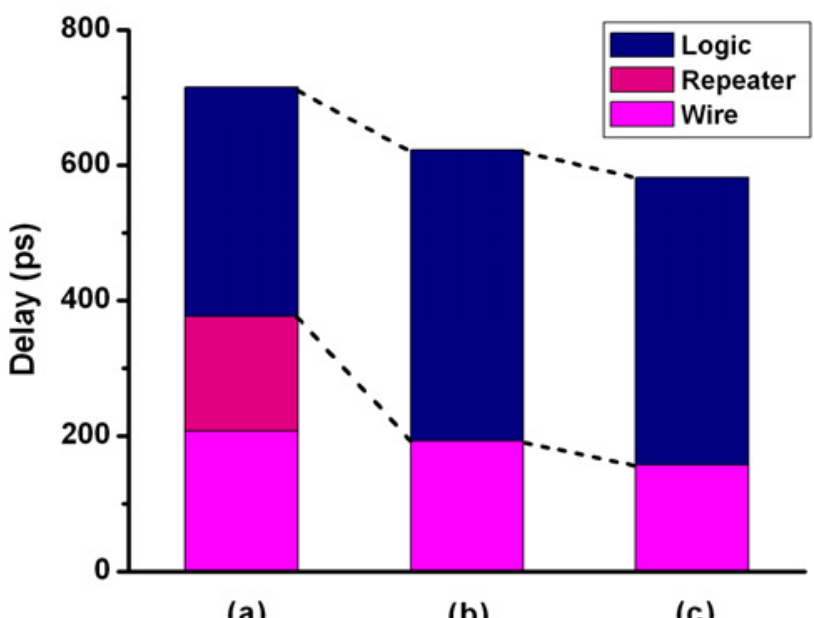

(a)

(b)

(c)

Figure 4: Delay breakdown (logic delay, repeater delay, and wire delay) of the three schemes in Figure 2 at iso-energy of 1.1pJ. 


\section{EVALUATING UTILITY OF LARGE CELLS IN TECHNOLOGY MAPPING}

To evaluate the utility of technology mapping for general circuits in scaled technologies, we compare pairs of libraries for several benchmarks with each technology. 'Original' scheme uses original standard cell library without any restriction, while 'No Large Cells' scheme is confined to the library where there are only 1-input and 2-input gates available.

\subsection{Methodology}

Figure 5 shows the flow chart for both approaches. Starting from the same behavioral netlist, logic synthesis (Synopsys Design Compiler 2007.03-sp2) is applied for each scheme with a restriction on the 'No Large Cells' scheme to use only 1-input or 2-input standard cells. After logic synthesis, the structural netlist goes through timing-driven placement, physical synthesis, and timing-driven routing (Cadence SoC Encounter 6.1.2). Post-placement logic restructuring is executed if necessary, but the restriction on the number of inputs of gates still holds in the 'No Large Cells' scheme. Finally, timing analysis is performed for both approaches with all back-end parasitics including coupling capacitance. This procedure was done for industrial $130 \mathrm{~nm}, 90 \mathrm{~nm}, 65 \mathrm{~nm}$, and $45 \mathrm{~nm}$ technologies, and benchmark circuits from IWLS 2005 [12] were used (s35932 from ISCAS family and the rest of them from OpenCores family). In the overall flow, the proposed scheme does not add any intermediate steps or iterations to the baseline. In fact, our approach seeks to reduce resource utilization (less standard cells from the library) while also improving delay.

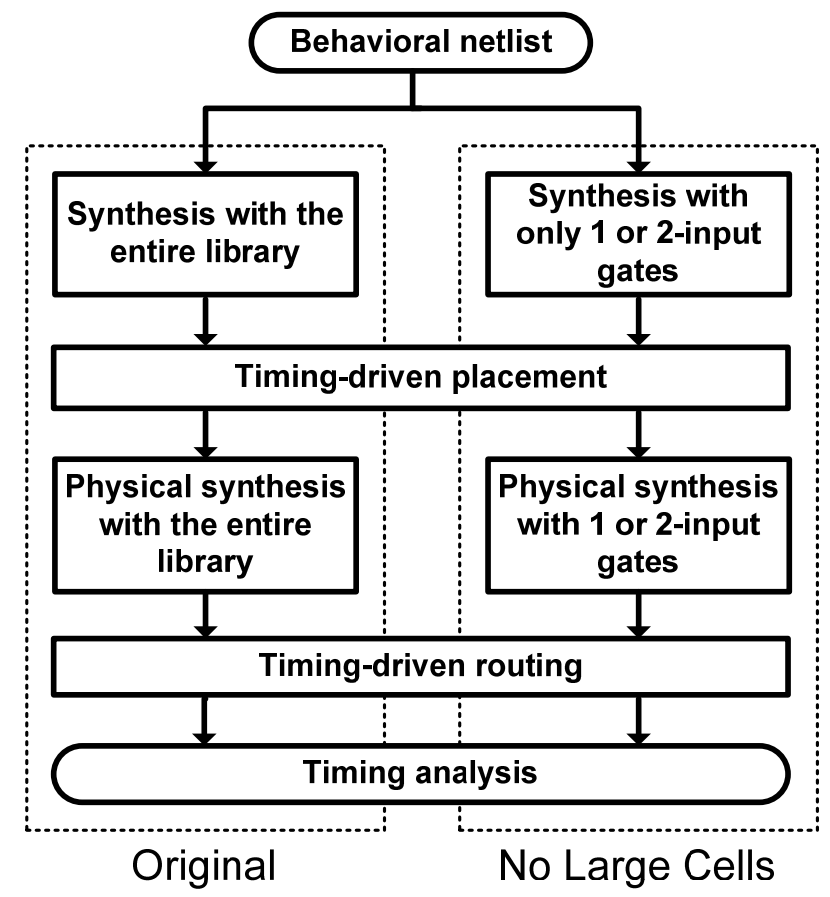

Figure 5: Flow chart for the methodology of 'Original' and 'No Large Cells'.

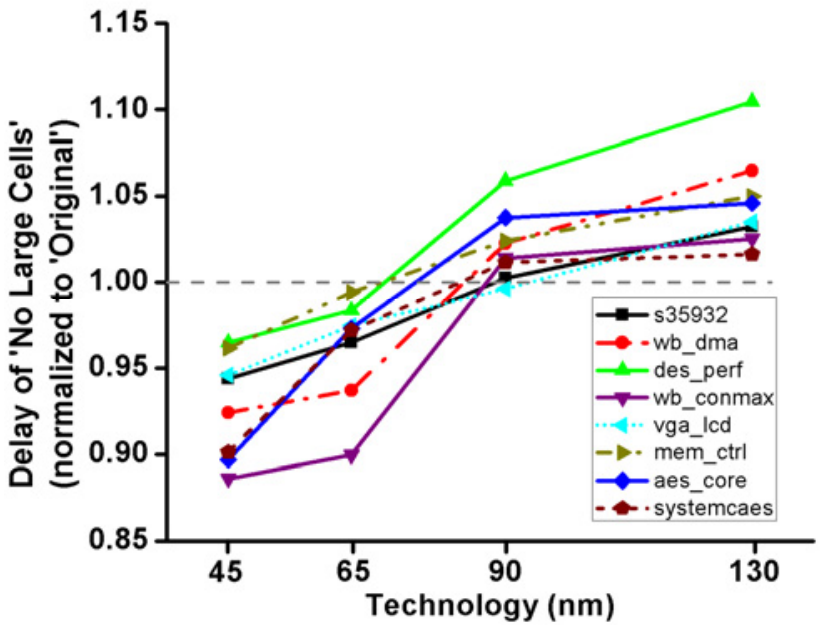

Figure 6: Critical path delay comparison of IWLS benchmarks using 'Original' and 'No Large Cells' approach in $130 \mathrm{~nm}, 90 \mathrm{~nm}, 65 \mathrm{~nm}$, and $45 \mathrm{~nm}$ technology.

\subsection{Experimental Results}

One expects the 'No Large Cells' approach to increase the gate count due to a more limited standard cell library. However, the critical path could actually benefit from more gates since both the wire capacitance and the number of required buffers are reduced.

Figure 6 compares the critical path delay between 'Original' and 'No Large Cells' configurations for eight benchmarks. Delay of 'No Large Cells' scheme is normalized to that of 'Original' scheme. The monotonic trend shown in Figure 6 illustrates the decreasing utility of large standard cells in technology mapping for more advanced technologies. At $65 \mathrm{~nm}$ and $45 \mathrm{~nm}$ technology, discarding large standard cells (3-inputs or more) gave better results (1-12\%) in critical path

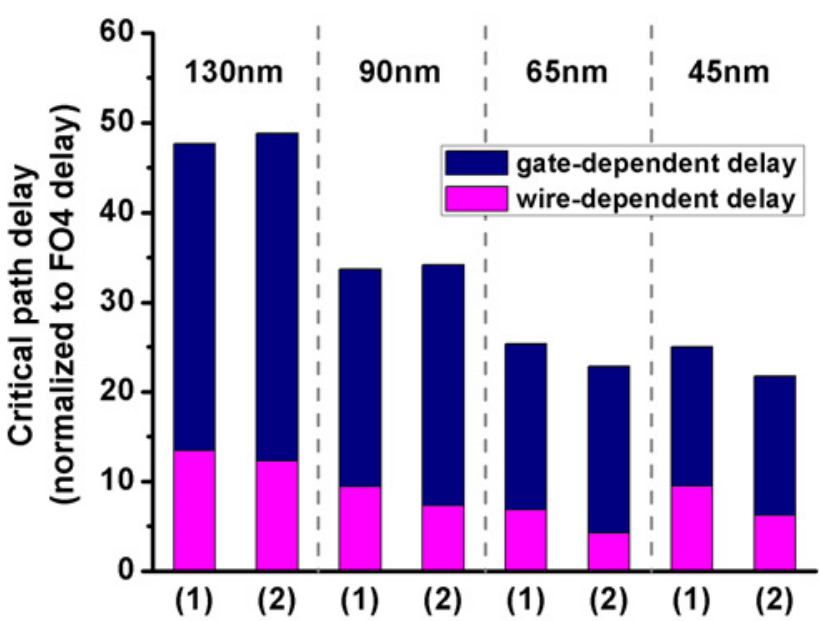

Figure 7: Critical path delay breakdown (gate-dependent delay and wire-dependent delay) of benchmark wb_conmax for (1) 'Original' and (2) 'No Large Cells' approach across four technology nodes. 
Table 1: Detailed comparison of the benchmarks for 'Original' and 'No Large Cells' scheme on critical path, average wire length (=total routed wire length/wire count), inserted buffer count, total standard cell count, wire capacitance, and total standard cell area is shown for (a) $65 \mathrm{~nm}$ and (b) $45 \mathrm{~nm}$ technology.

(a) $65 \mathrm{~nm}$

\begin{tabular}{|c|c|c|c|c|c|c|c|c|c|c|c|c|}
\hline \multirow{3}{*}{ Benchmark } & \multicolumn{6}{|c|}{ Original } & \multicolumn{6}{|c|}{ No Large Cells (vs. Original) } \\
\hline & \multirow{2}{*}{$\begin{array}{c}\text { Critical } \\
\text { path } \\
\text { delay } \\
\text { (FO4) }\end{array}$} & \multirow{2}{*}{$\begin{array}{l}\text { Avg. } \\
\text { wire } \\
\text { length } \\
(\mu \mathrm{m}) \\
\end{array}$} & \multirow{2}{*}{$\begin{array}{c}\text { Buffer } \\
\text { count / total } \\
\text { cell count }\end{array}$} & \multicolumn{2}{|c|}{$\begin{array}{c}\text { Wire capacitance } \\
\text { (fF) }\end{array}$} & \multirow{2}{*}{$\begin{array}{l}\text { Cell } \\
\text { area } \\
\left(\mu \mathrm{m}^{2}\right)\end{array}$} & \multirow{2}{*}{$\begin{array}{l}\text { Critical } \\
\text { path } \\
\text { delay }\end{array}$} & \multirow{2}{*}{$\begin{array}{l}\text { Avg. } \\
\text { wire } \\
\text { length }\end{array}$} & \multirow{2}{*}{$\begin{array}{l}\text { Buffer count } \\
/ \text { total cell } \\
\text { count }\end{array}$} & \multicolumn{2}{|c|}{ Wire capacitance } & \multirow{2}{*}{$\begin{array}{l}\text { Cell } \\
\text { area }\end{array}$} \\
\hline & & & & total & coupling & & & & & total & coupling & \\
\hline s35932 & 10.9 & 21.5 & $279 / 5764$ & 19.3 & 9.25 & 35512 & $-3.5 \%$ & $+3.4 \%$ & $-10 \% /+35 \%$ & $+14 \%$ & $+12 \%$ & $+10.6 \%$ \\
\hline wb_dma & 14.9 & 36.5 & $180 / 4968$ & 41.0 & 28.5 & 24069 & $-6.3 \%$ & $-15.8 \%$ & $-19 \% /+19 \%$ & $-5 \%$ & $-8 \%$ & $+4.0 \%$ \\
\hline des_perf & 20.1 & 23.7 & $511 / 69733$ & 244.8 & 128.2 & 320170 & $-1.7 \%$ & $-11.3 \%$ & $-44 \% /+12 \%$ & $-2 \%$ & $-4 \%$ & $-1.6 \%$ \\
\hline mem_ctrl & 21.8 & 28.1 & $217 / 5227$ & 22.9 & 12.2 & 50224 & $-0.6 \%$ & $-23.1 \%$ & $-27 \% /+37 \%$ & $+2 \%$ & $-1 \%$ & $+6.5 \%$ \\
\hline aes_core & 25.6 & 26.0 & $475 / 18568$ & 84.1 & 54.1 & 108502 & $-2.7 \%$ & $-22.9 \%$ & $-11 \% /+19 \%$ & $-15 \%$ & $-22 \%$ & $-9.8 \%$ \\
\hline systemcaes & 30.1 & 34.0 & $832 / 5678$ & 36.9 & 24.1 & 53441 & $-2.8 \%$ & $-19.0 \%$ & $-27 \% /+20 \%$ & $+7 \%$ & $+2 \%$ & $+20.9 \%$ \\
\hline Average & & & & & & & $-3.8 \%$ & $-19.7 \%$ & $-20 \% /+31 \%$ & $-4 \%$ & $-8 \%$ & $+7.7 \%$ \\
\hline
\end{tabular}

(b) $45 \mathrm{~nm}$

\begin{tabular}{|c|c|c|c|c|c|c|c|c|c|c|c|c|}
\hline \multirow{3}{*}{ Benchmark } & \multicolumn{6}{|c|}{ Original } & \multicolumn{6}{|c|}{ No Large Cells (vs. Original) } \\
\hline & \multirow{2}{*}{$\begin{array}{c}\text { Critical } \\
\text { path } \\
\text { delay } \\
\text { (FO4) }\end{array}$} & \multirow{2}{*}{$\begin{array}{l}\text { Avg. } \\
\text { wire } \\
\text { length } \\
(\mu \mathrm{m})\end{array}$} & \multirow{2}{*}{$\begin{array}{c}\text { Buffer } \\
\text { count / total } \\
\text { cell count }\end{array}$} & \multicolumn{2}{|c|}{$\begin{array}{c}\text { Wire capacitance } \\
\text { (fF) }\end{array}$} & \multirow{2}{*}{$\begin{array}{c}\text { Cell } \\
\text { area } \\
\left(\mu \mathrm{m}^{2}\right)\end{array}$} & \multirow{2}{*}{$\begin{array}{c}\text { Critical } \\
\text { path } \\
\text { delay }\end{array}$} & \multirow{2}{*}{$\begin{array}{l}\text { Avg. } \\
\text { wire } \\
\text { length }\end{array}$} & \multirow{2}{*}{$\begin{array}{l}\text { Buffer count } \\
/ \text { total cell } \\
\text { count }\end{array}$} & \multicolumn{2}{|c|}{ Wire capacitance } & \multirow{2}{*}{$\begin{array}{l}\text { Cell } \\
\text { area }\end{array}$} \\
\hline & & & & total & coupling & & & & & total & coupling & \\
\hline s35932 & 12.6 & 14.0 & $283 / 7155$ & 14.3 & 7.2 & 20131 & $-5.6 \%$ & $-14.5 \%$ & $-5.3 \% /+18 \%$ & $+8 \%$ & $+7 \%$ & $+7.5 \%$ \\
\hline wb_dma & 15.7 & 27.3 & $268 / 6109$ & 27.8 & 17.7 & 13600 & $-7.7 \%$ & $-12.5 \%$ & $-13 \% /+6 \%$ & $-9 \%$ & $-12 \%$ & $-1.7 \%$ \\
\hline des_perf & 23.3 & 17.3 & $1599 / 85297$ & 213.9 & 122.3 & 186916 & $-3.5 \%$ & $-16.7 \%$ & $-27 \% /+20 \%$ & $-4 \%$ & $-10 \%$ & $+3.1 \%$ \\
\hline wb_conmax & 25.0 & 46.3 & 1874 / 27280 & 256.2 & 231.0 & 55171 & $-12.3 \%$ & $-37.2 \%$ & $-256 \% /+70 \%$ & $-21 \%$ & $-29 \%$ & $+20.1 \%$ \\
\hline vga_lcd & 31.7 & 28.6 & 3807 / 42890 & 183.1 & 108.2 & 153413 & $-5.4 \%$ & $-20.6 \%$ & $-5 \% /+28 \%$ & $-2 \%$ & $-4 \%$ & $+8.8 \%$ \\
\hline mem_ctrl & 24.3 & 20.3 & $307 / 6285$ & 20.7 & 12.7 & 14733 & $-3.9 \%$ & $-23.4 \%$ & $-11 \% /+32 \%$ & $-4 \%$ & $-10 \%$ & $+7.2 \%$ \\
\hline aes_core & 26.2 & 18.9 & $1329 / 17485$ & 63.4 & 42.6 & 46640 & $-10.3 \%$ & $-19.1 \%$ & $-54 \% /+39 \%$ & $-7 \%$ & $-4 \%$ & $+8.4 \%$ \\
\hline systemcaes & 30.4 & 26.4 & $1031 / 6557$ & 32.6 & 21.1 & 15274 & $-9.9 \%$ & $-22.2 \%$ & $-36 \% /+16 \%$ & $-7 \%$ & $-2 \%$ & $+15.7 \%$ \\
\hline Average & & & & & & & $-7.3 \%$ & $-17.2 \%$ & $-22 \% /+29 \%$ & $-6 \%$ & $-8 \%$ & $+8.6 \%$ \\
\hline
\end{tabular}

delay than the original technology mapping for all benchmarks. Breaking up the wire into more segments proves to be effective at $65 \mathrm{~nm}$ and below through reducing the wire delay components. The delay breakdown for benchmark wb conmax is shown in Figure 7 across four technology nodes. Gate-dependent delay is defined as the sum of intrinsic gate delay and gate load delay, which is basically the circuit delay when no wire is present. Wiredependent delay consists of inserted buffer delay and wire load delay, which are the delay elements generated due to routed wires. It can be seen that our approach increases gatedependent delay by a minimal amount, but the wiredependent delay component is reduced significantly $(35 \%$ in $45 \mathrm{~nm}$ ), leading to an overall $12 \%$ performance improvement in $45 \mathrm{~nm}$ node. Note that the relative portion of wiredependent delay grew considerably at the $45 \mathrm{~nm}$ node. This is mostly due to the sharp increase of resistance of minimum width wires in $45 \mathrm{~nm}$, considering that the capacitance of a unit length wire does not change significantly for each technology step.
Table 1 shows a detailed comparison on several metrics for eight benchmarks for both $65 \mathrm{~nm}$ and $45 \mathrm{~nm}$ technology to check whether the 'No Large Cells' approach is working as proposed. Typically a large number of buffers are inserted during timing optimization for the given benchmark circuits, and the number of buffers is reduced by $5-54 \%$ by breaking the long wires into short wires with more gates. Average wire length and wire capacitance (both total and coupling) show noticeable reduction except for the relatively small s35932 benchmark. The reduction in coupling capacitance is more than that in ground capacitance, which is due to the observed higher routing congestion in intermediate and high metal layers in the 'Original' configuration leading to increased coupling capacitance. This fact is encouraging because coupling capacitance increasingly dominates the overall wire capacitance with technology scaling. In the s35932 benchmark, the fact that the critical path delay marginally decreased despite an increase in wire length and capacitance suggests further improvement by introducing the proposed approach only on timing-critical nets. Benchmark 


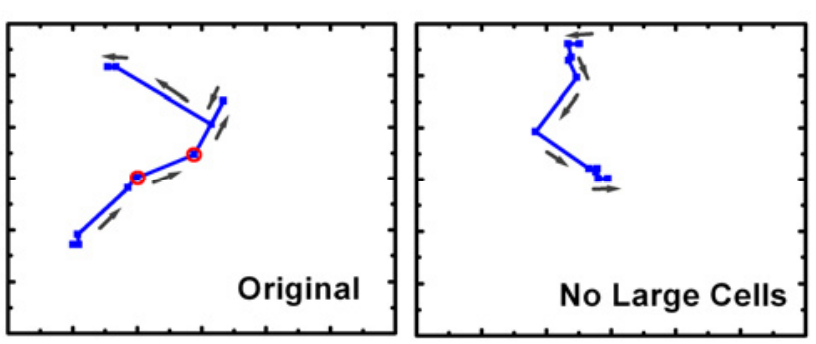

(a) Benchmark wb_dma at $65 \mathrm{~nm}$ node

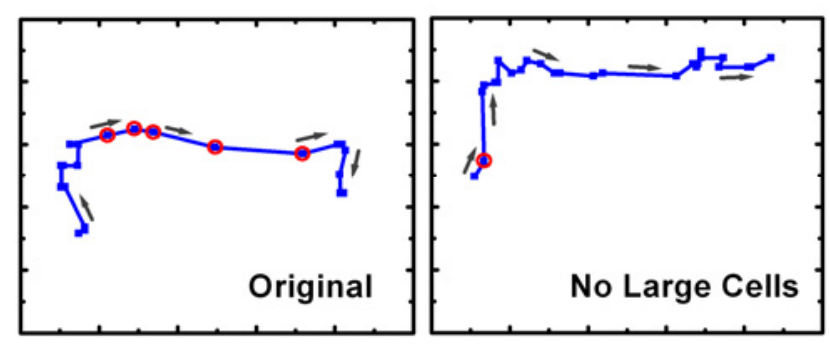

(b) Benchmark systemcaes at $45 \mathrm{~nm}$ node

Figure 8: Critical path comparison between 'Original' and 'No Large Cells' configuration for benchmarks (a) wb_dma at $65 \mathrm{~nm}$ technology node and (b) systemcaes at $\overline{45} \mathrm{~nm}$ technology node is shown (dots with circles represent inserted buffers).

des 3 _perf shows a small improvement in critical path delay in spite of a large reduction in the buffer count, especially in $65 \mathrm{~nm}$, because the inserted buffer count is a small portion of the total standard cell count $(0.7 \%)$.

It is not surprising that the standard cell count is increased by $12-54 \%$, but the standard cell area overhead is only $8.6 \%$ on average at $45 \mathrm{~nm}$ technology since 1 -input and 2-input gates are typically smaller than complex gates. This area increase would not necessarily result in comparable die area increases in modern microprocessors or SoC designs because embedded memories and hard IP blocks consume a large portion of the total chip area, making standard cell area a relatively lesser concern [13-14]. Also, in designs with hierarchical floorplans, increasing the area of one partition does not affect the area of the entire chip, and designs requiring high I/O bandwidth (such as network processors) are pad-limited. Furthermore, by expanding this work to remove large cells only from timing critical paths, similar delay results with much smaller area increases are expected since standard cells on critical paths are responsible for only a small fraction of overall cell area.

The critical paths and signal directions of benchmarks wb_dma (65nm node) and systemcaes ( $45 \mathrm{~nm}$ node) for configurations 'Original' and 'No Large Cells' are visualized in Figure 8. For benchmark wb_dma, the path is noticeably shorter, has fewer long wires and no inserted buffers in the 'No Large Cells' configuration, yielding an improvement of $6.3 \%$ in critical path delay. Benchmark systemcaes in $45 \mathrm{~nm}$ node is also a good example of effectively converting wire delay into gate delay. In the 'Original' case, five buffers are
Table 2: Dynamic and leakage power comparison between 'Original' and 'No Large Cells' scheme for (a) $65 \mathrm{~nm}$ and (b) $45 \mathrm{~nm}$ technology.

(a) $65 \mathrm{~nm}$

\begin{tabular}{|c|c|c|c|c|}
\hline \multirow{2}{*}{ Benchmark } & \multicolumn{2}{|c|}{ Dynamic power } & \multicolumn{2}{c|}{ Leakage power } \\
\cline { 2 - 5 } & $\begin{array}{c}\text { Original } \\
(\mathrm{mW})\end{array}$ & $\begin{array}{c}\text { No } \\
\text { Large } \\
\text { Cells }\end{array}$ & $\begin{array}{c}\text { Original } \\
(\mu \mathrm{W})\end{array}$ & $\begin{array}{c}\text { No } \\
\text { Large } \\
\text { Cells }\end{array}$ \\
\hline s35932 & 5.3 & $+0.6 \%$ & 47.2 & $+14.8 \%$ \\
\hline wb_dma & 2.8 & $+1.1 \%$ & 34.1 & $+5.9 \%$ \\
\hline des_perf & 26.4 & $-4.5 \%$ & 448.4 & $0 \%$ \\
\hline wb_conmax & 11.7 & $-7.9 \%$ & 145.3 & $+22.7 \%$ \\
\hline vga_lcd & 22.6 & $+7.1 \%$ & 408.1 & $+11.5 \%$ \\
\hline mem_ctrl & 2.4 & $+0.5 \%$ & 35.3 & $+11.3 \%$ \\
\hline aes_core & 9.4 & $-9.0 \%$ & 95.6 & $+2.3 \%$ \\
\hline systemcaes & 4.0 & $+6.5 \%$ & 37.9 & $+25.2 \%$ \\
\hline Average & & $-0.7 \%$ & & $+11.7 \%$ \\
\hline
\end{tabular}

(b) $45 \mathrm{~nm}$

\begin{tabular}{|c|c|c|c|c|}
\hline \multirow{2}{*}{ Benchmark } & \multicolumn{2}{|c|}{ Dynamic power } & \multicolumn{2}{c|}{ Leakage power } \\
\cline { 2 - 5 } & $\begin{array}{c}\text { Original } \\
(\mathrm{mW})\end{array}$ & $\begin{array}{c}\text { No } \\
\text { Large } \\
\text { Cells }\end{array}$ & $\begin{array}{c}\text { Original } \\
(\mu \mathrm{W})\end{array}$ & $\begin{array}{c}\text { No } \\
\text { Large } \\
\text { Cells }\end{array}$ \\
\hline s35932 & 2.7 & $+1.2 \%$ & 67.9 & $+20.0 \%$ \\
\hline wb_dma & 1.3 & $+5.1 \%$ & 48.1 & $+0.3 \%$ \\
\hline des_perf & 11.9 & $+0.4 \%$ & 706.2 & $+6.1 \%$ \\
\hline wb_conmax & 10.3 & $-5.4 \%$ & 242.8 & $+22.6 \%$ \\
\hline vga_lcd & 9.4 & $+4.8 \%$ & 460.1 & $+7.0 \%$ \\
\hline mem_ctrl & 1.4 & $+1.5 \%$ & 45.9 & $+14.6 \%$ \\
\hline aes_core & 3.6 & $+2.1 \%$ & 162.7 & $+12.7 \%$ \\
\hline systemcaes & 1.8 & $-4.4 \%$ & 59.4 & $+23.7 \%$ \\
\hline Average & & $+0.5 \%$ & & $+13.4 \%$ \\
\hline
\end{tabular}

inserted to send the signal to the distant location, whereas a number of small standard cells are spread out to serve as a repeater while also performing logic operation in the "No Large Cells' case.

In addition to circuit performance, power consumption is considered in our analysis. Table 2 shows both dynamic and leakage power consumption of the final netlists for the two schemes in $65 \mathrm{~nm}$ technology. We used randomized switching data with average activity factor of 0.2 for each benchmark, and measured power using Synopsys NanoSim. For a few benchmarks, power consumption of the 'No Large Cells' scheme is actually lower than that of the 'Original' scheme, due to the interaction of the appreciably lower buffer count and smaller wire capacitance. For the vga_lcd benchmark, buffer count is not significantly reduced by the simplified technology mapping, resulting in $7.1 \%$ and $4.8 \%$ power increase in $65 \mathrm{~nm}$ and $45 \mathrm{~nm}$, respectively. The power overhead for the wb_dma, mem_ctrl, and s35932 benchmarks is insignificant. Overall, despite the increased gate count, the capacitance of 1-input and 2-input gates is 
small, leading to comparable overall power consumption of the 'No Large Cells' scheme as that of the 'Original' scheme.

The leakage power overhead in Table 2 is largely proportional to the standard cell area increase in Table 1. More precisely, the reason why the leakage overhead is slightly larger than the area overhead is that small standard cells have shorter stacks of transistors leading to less stack effect and more leakage power. However, the additional leakage power is relatively small $(\sim 1 / 100$ of dynamic power in all benchmarks) and the net effect on total power as seen in Table 2 is very low for these typical high-performance designs.

Our results on full integrated circuits motivate placementaware technology mapping and post-placement logic restructuring, which can indeed improve timing. However, commercial tools available to us only partially include this feature, and in its absence, we demonstrate that large standard cells are not particularly useful on critical paths. The arguments from Section 2 suggest that even with placement-driven technology mapping and post-placement logic restructuring, large cells will be less useful on critical paths. An additional advantage of our approach is that breaking down large cells into smaller ones improves routability by enhancing the ability to reduce routing congestion [15-16].

Throughout these benchmark experiments for critical path delay optimization, we execute synthesis, placement, and routing for the same circuit. As a result the size of the circuit and the length of long wires will also decrease for each technology step, which is why the wire-dependent delay in the 'Original' approach in Figure 7 decreases at each technology node from $130 \mathrm{~nm}$ to $65 \mathrm{~nm}$. However, when technology scaling is used to double the number of on-chip transistors, the chip size and longest wires do not shrink. If technology mapping is skipped under this assumption (higher levels of integration for scaled technologies), wire delay will dominate due to inter-module communication and we suggest that the performance improvement using the proposed approach would increase.

\section{CONCLUSION}

Our work offers a first-of-a-kind careful analysis of technology mapping across four technology nodes. While this step has been commonly used in logic synthesis flows, we point out that the use of large standard cells in it appears unnecessary and even harmful for high-performance designs at $65 \mathrm{~nm}$ and below (low power designs could still benefit from technology mapping through reduced leakage). This is a consequence of uneven scaling of wire and gate delay, as well as the fact that technology mapping essentially trades gate counts for an increased number of long wires (as shown in Table 1). Empirical trends observed for large benchmark circuits mapped to $130 \mathrm{~nm}, 90 \mathrm{~nm}, 65 \mathrm{~nm}$, and $45 \mathrm{~nm}$ libraries suggest that the $65 \mathrm{~nm}$ node is an inflection point for the utility of large cells in technology mapping.

\section{REFERENCES}

[1] A. Abdollahi, and M. Pedram, "A new canonical form for fast boolean matching in logic synthesis and verification," Proc. Design Automation Conference, pp. 379-384, 2005.

[2] G. Agosta, et. al., "A unified approach to canonical formbased boolean matching," Proc. Design Automation Conference, pp. 841-846, 2007.

[3] D. Sylvester, and K. Keutzer, "Getting to the bottom of deep submicron II: a global wiring paradigm," Proc. International Symposium on Physical Design, pp. 193-200, 1999.

[4] P. Saxena, et. al., "Repeater scaling and its impact on CAD," IEEE Transactions of Computer-Aided Design of Integrated Circuits and Systems, Vol. 23, pp. 451-463, 2004.

[5] C. Alpert, et. al., "Buffer insertion with accurate gate and interconnect delay computation," Proc. Design Automation Conference, pp. 479-484, 1999.

[6] Y. Ismail, and E. Friedman, "Optimum repeater insertion based on a CMOS delay model for on-chip RLC interconnect," Proc. International ASIC Conference, pp. 369-373, 1998

[7] A. Nalamalpu, and W. Burleson, "Repeater insertion in deep sub-micron CMOS: ramp-based analytical model and placement sensitivity analysis," Proc. International Symposium on Circuits and Systems, pp. 766-799, 2000.

[8] R. Otten, and R. Brayton, "Planning for performance," Proc. Design Automation Conference, pp. 122-127, 1998.

[9] M. Pedram, and N. Bhat, "Layout driven technology mapping," Proc. Design Automation Conference, pp. 99105, 1991.

[10] A. Lu, et. al., "Combining technology mapping with postplacement resynthesis for performance optimization," Proc. International Conference on Computer Design, pp. 616$621,1998$.

[11] G. Stenz, et. al., "Performance optimization by interacting netlist transformations and placement," IEEE Transactions on Computer-Aided Design of Integrated Circuits and Systems, pp. 350-358, Vol. 19, No. 3, March 2000.

[12] IWLS 2005 benchmarks. http://iwls.org/iwls2005/benchmarks.html.

[13] E. Wein, and J. Benkoski, "Hard macros will revolutionize SoC Design," EE Design, August 2004. http://www.eetimes.com/showArticle.jhtml?articleID=2680 7055 .

[14] T. Chen, et. al., "MP-trees: a packaging-based macroplacement algorithm for mixed-size designs," Proc. Design Automation Conference, pp. 447-452, 2007.

[15] S. Plaza, I. Markov, and V. Bertacco, "Optimizing nonmonotonic interconnect using functional simulation and logic restructuring," Proc. of ISPD, pp. 95-102, 2008.

[16] R. Shelar, P. Saxena, X. Wang, and S. Sapatnekar, "An efficient technology mapping algorithm targeting routing congestion under delay constraints," Proc. of ISPD, pp. 137-144, 2005. 